FORMATION Formation emploi

Revue française de sciences sociales

108 | octobre-décembre 2009

Les processus de professionnalisation

\title{
Fermetures d'usines : les associations d'anciens salariés comme espaces de médiation originaux.
}

Werksschliessungen: Verbände ehemaliger Beschäftigter als neue

Vermittlungsräume

Factory closures: associations of former employees as new areas for mediation

Cierre de fabricas : las asociaciones de ex asalariados como espacios de

mediacion originales

Delphine Corteel

\section{(2) OpenEdition}

Journals

Édition électronique

URL : http://journals.openedition.org/formationemploi/2086

DOI : 10.4000/formationemploi.2086

ISSN : 2107-0946

Éditeur

La Documentation française

Édition imprimée

Date de publication : 1 décembre 2009

Pagination : 53-65

ISSN : 0759-6340

Référence électronique

Delphine Corteel, «Fermetures d'usines : les associations d'anciens salariés comme espaces de médiation originaux. », Formation emploi [En ligne], 108 | octobre-décembre 2009, mis en ligne le 01 janvier 2012, consulté le 30 octobre 2020. URL : http://journals.openedition.org/formationemploi/ 2086 ; DOI : https://doi.org/10.4000/formationemploi.2086 


\section{DOSSIER}

\section{Fermetures d'usines : les associations d'anciens salariés comme espaces de médiation originaux}

Delphine Corteel*

Là où les cellules de reclassement invitent souvent à tourner la page, l'association d'anciens salariés confère un sens et une utilité nouvelle à l'expérience collective et à la profession disparue, susceptibles de permettre la poursuite des parcours professionnels.

Depuis quelques années, les fermetures d'usines et les luttes que mènent leurs (anciens) salariés à cette occasion font l'objet d'une grande attention, tant dans les sciences sociales que chez les cinéastes, les écrivains ou les journalistes. Au-delà de la rupture du lien économique et juridique entre employeurs et salariés, c'est un ensemble complexe de liens sociaux entre salariés, entre générations, entre l'usine et son territoire qui sont bousculés et dont la dislocation malmène les personnes (Linhart et al., 2002). Cet événement prend une acuité particulière dans des régions comme les Ardennes ou le Nord-Pas-deCalais où tous les secteurs industriels - la métallurgie, le textile, les mines...- qui ont marqué l'histoire et l'identité territoriale périssent. On assiste alors à la disparition d'un "monde de production» (Salais et Storper, 1993), porteur d'une identité professionnelle forte qui conférait un sens et une utilité sociale aux savoirs et savoir-faire transmis de génération en génération et dont les personnes licenciées sont les dernières dépositaires. Comme le souligne Hughes, "le métier d'un homme est l'une des composantes les plus importantes de son identité sociale, de son moi, et même de son destin dans son unique existence » (1997, p. 76).

* Delphine Corteel est maître de conférences en sociologie (université de Reims Champagne-Ardenne) et chercheure au Laboratoire Institutions et dynamiques historiques de l'économie (Cachan). Elle contribue notamment au projet ANR Expertise sur autrui autour des critères et des pratiques des processus de décision des " experts sur autrui ", dans le cadre des contrats de transition professionnelle.

Elle a publié notamment : Corteel Delphine (2009), « Lutter pour la reconversion d'un site industriel et la dignité des hommes qui l'ont fait vivre. Analyse d'expériences menées par les ex-salariés de Metaleurop-Nord. ", in Causer JeanYves, Durand Jean-Pierre et Gasparini William (dirs.), Les identités au travail. Analyses et controverses, Toulouse, Octarès, pp. 113-122. Corteel Delphine, Zimmermann Bénédicte (2007), "Capacités et développement professionnel », Formation Emploi, n 98, avril-juin. 
La fermeture, événement biographique traumatique partagé par les anciens salariés, pose la question de l'avenir - les destins individuels possibles - et du passé - sa signification. En effet, "l'irruption d'un évènement marquant [...] permet ou oblige à une réélaboration éventuelle du passé» (Leclerc-Olive, 1998, p. 103). La fermeture comme événement a ceci de particulier qu'elle affecte non seulement les personnes mais aussi le collectif : le collectif objectif de l'ensemble des salariés de l'usine concernée et surtout le collectif subjectif ou collectif professionnel investi quotidiennement dans la "recréation de l'organisation du travail » (Clot, 2003, p. 1). Ainsi la réélaboration du passé porte aussi sur le sens et la valeur de ce collectif, en particulier dans la perspective de la poursuite du parcours professionnel, c'està-dire de l'inscription possible dans un nouveau collectif professionnel.

Comment dès lors accompagner les salariés touchés par la fermeture ? Depuis le milieu des années 80 , les politiques publiques de traitement du chômage s'inscrivent dans la logique du reclassement, celle du retour à l'emploi le plus rapide possible, c'est-à-dire le rétablissement d'un lien économique et juridique avec un employeur. Sont considérées comme reclassées, c'est-à-dire titulaires d'un emploi stable, toutes les personnes embauchées en CDI (contrat à durée indéterminée) certes, mais aussi en CDD (contrat à durée déterminée) et en mission d'intérim de plus de six mois. Dans la logique du reclassement, seule la dimension de l'emploi est prise en compte et la qualité de ce dernier est évaluée sur des critères somme toute assez pauvres. L'individualisation du traitement $\mathrm{du}$ chômage entraîne dans son sillage une certaine psychologisation de la prise en charge qui prend en compte tant bien que mal la souffrance morale et psychologique des individus confrontés au licenciement. En mettant l'accent sur la responsabilité individuelle et l'employabilité, elle n'est pas sans fragiliser les personnes, faute de leur donner les véritables moyens d'exercer leur responsabilité (Corteel, Zimmermann, 2006). Quoiqu'il en soit, la dimension $\mathrm{du}$ travail, de son contenu, des liens sociaux et du collectif professionnel reste quasiment impensée. Quant au passé, il est souvent écarté ; comme le préconisent certains consultants des cellules de reclassement, il faut «couper le cordon ombilical avec l'entreprise » (Tuchszirer, 2005, p. 164)», «faire le deuil», ou encore «tourner la page». Le passé et l'expérience collective sont présentés comme des obstacles pour l'avenir, venant ajouter au sentiment d'inutilité insufflé par la fermeture. Or, en même temps qu'on demande aux salariés d'oublier, on leur demande de se souvenir pour identifier des compétences, rédiger des curriculum vitae, des dossiers de demande de formation ou de Validation des acquis de l'expérience. Injonctions contradictoires, difficilement tenables. Pourtant, c'est bien la poursuite d'un parcours professionnel et, plus largement, d'un parcours de vie qu'il convient d'envisager. Comment puiser dans le passé - collectif - les ressources nécessaires pour l'avenir - individuel ? Comment examiner le passé, le travailler, sans sombrer dans une nostalgie indépassable? Cet article examine les enjeux de la poursuite des parcours professionnels - poursuite que l'on pourrait envisager comme une re-professionnalisation - et en étudie le caractère problématique sous l'angle des rapports au passé du travail à l'usine et au collectif professionnel. Pour ce faire est explorée la piste ouverte par l'expérience originale du travail d'accompagnement réalisé par l'association des anciens salariés de Metaleurop-Nord. Cette association ne se substitue pas aux professionnels du reclassement, elle travaille avec eux. Elle réinvente le collectif de travail et le mobilise pour soutenir les projets professionnels des anciens salariés. Ce faisant, elle permet précisément de redonner un sens et une utilité à la profession aujourd'hui disparue, de réactiver les réseaux et les liens constitués au moment où l'usine fonctionnait, au service des avenirs professionnels de chacun de ceux qui la sollicitent.

Dans une première partie, nous reviendrons sur le sens que les salariés confrontés à la fermeture de leur usine donnent à cet événement. Metaleurop, victime d'une mise en faillite délibérée et abandonnée par sa maison mère (Gaulin, 2005), est un cas un peu particulier. Afin de dépasser cette spécificité, sont utilisés non seulement les entretiens et observations réalisés sur ce cas, mais on mobilise également des ouvrages portant sur quatre autres cas de fermetures survenues en France, à la fin des années 90 ou au début des années 2000, dans le secteur industriel (voir encadré 1).

Ainsi, pour Metaleurop et les autres cas étudiés, on peut analyser la fermeture comme la fin d'une 


\section{Encadré 1 \\ Sources et méthode}

Ce travail anthropologique s'appuie sur deux grands types de sources:

- Des matériaux recueillis lors de cinq missions de terrain réalisées entre octobre 2006 et juin 2007, sur le site de Metaleurop-Nord, alliant observations et entretiens. Une vingtaine d'entretiens approfondis ont été conduits avec des ex-salariés (comme ils se nomment eux-mêmes) très actifs pendant la lutte et engagés dans l'association des ex-salariés de Metaleurop-Nord, baptisée "Chœurs de fondeurs ( $^{*}$ ). Nous nous appuyons également sur un livre d'entretiens réalisés auprès des ouvriers, écrit par Frédéric Fajardie, sur un montage vidéo réalisé par les salariés eux-mêmes pour retracer leur aventure, intitulé "Corps de fondeurs » et diffusé à l'occasion d'une exposition consacrée à l'usine, qu'ils ont organisée au printemps 2006, ainsi que sur le film Le conflit Metaleurop, réalisé par un enfant d'un ex-salarié de l'usine.

- Les travaux d'autres chercheurs, auxquels sont attribués le statut de "monographies de fermeture ", en particulier la thèse de Manuella Roupnel-Fuentes (2007) qui porte sur le licenciement massif de salariés de plusieurs usines Moulinex en Normandie, le travail de Yannick Le Quentrec et Stéphanie Benson (2005) qui sont intervenues, à leur demande, auprès des anciens salariés de l'usine Job lfabricant de papier) ou l'enquête de Sylvie Malsan (2001) sur la fermeture d'Alcatel ( $\left.{ }^{* *}\right)$. Je m'appuie également sur le livre rédigé par Sylvain Rossignol (2008) à la demande des salariés de Roussel-Uclaf au moment de la fermeture de leur usine.

$\left(^{*}\right)$ : Cette enquête a été financée grâce au bonus qualité-recherche de l'université de Paris 8 et a été conduite conjointement par Delphine Corteel, Anne Duhin et Judith Hayem.

$(* *)$ : C'est certes un usage réducteur car tous ces travaux sont bien plus que des monographies et ils ont fortement stimulé la réflexion autour des questions traitées ici.

profession. Nous présenterons ensuite l'association des anciens salariés de Metaleurop, et son ancrage dans la profession disparue, dont elle mobilise et réactualise les valeurs, sera souligné. Dans une troisième partie, seront étudiées les pratiques de l'association en matière d'accompagnement professionnel des ex-salariés afin de dégager la spécificité de l'intermédiation proposée par cet acteur inhabituel sur le marché du travail. En conclusion, je reviendrai sur les enseignements que l'on peut tirer de cette expérience qui, bien que rare, n'est pas isolée. En effet, pour n'en citer que quelques-uns, chez Job ou chez Moulinex aussi les anciens salariés s'organisent en association, se mobilisent et investissent collectivement la question de leur avenir (professionnel) aux côtés des professionnels du reclassement ${ }^{1}$.

\footnotetext{
${ }^{1}$ Pour un aperçu du nombre et des activités des associations d'anciens salariés créées à la suite de fermetures, voir Fermetures de boîtes... et après?, édité par la Maison des Hommes et des Techniques de Nantes (2007).
}

\section{LA FERMETURE DE L'USINE... OU LA FIN D'UNE PROFESSION}

Fondeurs, ouvrières de production, papetiers, laborantins officiant dans des secteurs ou des métiers dont le prestige social varie considérablement selon les situations, les salariés confrontés à la fermeture de leur usine ont en commun de l'identifier comme la fin d'une profession «dans son usage ancien et le plus restreint», c'est-à-dire la fin de «métiers très prestigieux et qualifiés dont les membres agissaient pour le profit d'autrui» (Hughes, 1996, p. 63). Les salariés de Roussel-Uclaf demandent à Sylvain Rossignol d'écrire "un livre qui parle de cette chaîne de métiers qui contribuent à la recherche de médicaments innovants; un livre porteur d'espoir qui raconte l'aventure de ceux qui défendent leur travail parce qu'ils le savent utile et 
nécessaire $»^{2}$ (2008, p. 414). Les salariés de Metaleurop rappellent quant à eux que les produits qu'ils fabriquaient ont contribué aux technologies les plus avancées et sont utilisés quotidiennement par tout un chacun: "Metaleurop producteur recycleur. PLOMB pour nos batteries automobiles. ZINC pour l'anticorrosion de nos voitures et de nos immeubles. INDIUM pour communiquer sur tous nos écrans plats. GERMANIUM pour télécommuniquer sur nos câbles et nos fibres optiques. » (Corps de fondeurs). De même, Benson et Le Quentrec soulignent que «les salariés de Job ne travaillent pas dans n'importe quelle usine de production de masse. Ils fabriquent, aiment-ils à le répéter, le plus beau papier du monde et s'inscrivent à ce titre dans une longue lignée de papetiers qui ont en commun l'amour du produit et du travail bien fait. » (2005, p. 162). Et même dans une usine « de production de masse " comme chez Moulinex, on retrouve ce motif: "Il se dégage des personnes interviewées, la fierté d'avoir appartenu à une célèbre entreprise, à un groupe industriel connu à travers le monde, à une entreprise dans l'air du temps. L'appareil électroménager est un objet d'achat personnel et un objet à offrir. » (Roupnel-Fuentes, 2007, p. 171). Roussel-Uclaf, Metaleurop, Job, Moulinex, tous évoquent, d'une manière ou d'une autre, la fierté de leur métier, les savoir-faire pointus mis en œuvre, la grande qualité des produits et leur utilité sociale.

Du point de vue des salariés, la fermeture peut donc se lire comme la consécration paradoxale d'une profession. C'est au moment de son extinction que les salariés la revendiquent peut-être le plus fortement. Ainsi, au moment de la fermeture, le collectif se réinvente et se ressoude autour d'un produit (le papier, chez Job ; les médicaments chez Roussel-Uclaf), d'un métier (fondeur, chez Metaleurop) ou encore du nom de la marque (chez Moulinex ou Alcatel). La fermeture est alors identifiée comme la fin d'une profession. Elle représente la remise en cause de la « licence », au sens de l'autorisation légale d'exercer un métier, la négation de la spécificité des savoir-faire qui y sont associés et du collectif qui les a construits, enrichis, mis en œuvre. Elle signe également la fin du «mandat»,

${ }^{2}$ Cette citation est tirée de la postface du livre qui en explique la genèse et est signée de la présidente de l'association RU, association des anciens salariés de Roussel-Uclaf. c'est-à-dire la fin du contrôle des conditions de travail, de la transmission des savoir-faire et la fin de la possibilité de "fixer les termes selon lesquels il convient de penser un aspect particulier de la société, de la vie et de la nature » (Hughes, op. cit., p. 109). $\mathrm{Ce}$ point est particulièrement prégnant autour de Noyelles-Godault où est implantée Metaleurop. Le discours des riverains sur l'usine polluante, dangereuse, porteuse de maladie et de mort est venu supplanter celui sur l'usine créatrice de richesse, garantissant l'avenir de la région, porté par les salariés. Les salariés cherchent alors à défendre leur professionnalité, leur qualification, leur engagement et leur prestige, mis à mal par la fermeture. Les parcours professionnels envisageables après la fermeture s'avèrent problématiques dans la mesure où la profession disparaît et avec elle le contexte qui rendait lisible l'utilité sociale des savoir-faire, des qualifications et des compétences dont les salariés licenciés sont collectivement les dépositaires.

Pour que s'ouvre une nouvelle séquence du parcours professionnel, faut-il impérativement rompre avec l'ancien collectif et avec la profession ? La profession et l'expérience collective qu'elle a forgée peuventelles apporter un soutien spécifique et efficace à ses membres?

\section{DES « PROFESSIONNELS DE L'USINE » AU SERVICE DE LEURS COLLÈGUES}

Les associations d'anciens salariés mobilisent la profession et la font (re)vivre pour permettre, dans la mesure du possible, aux ex-salariés de construire une nouvelle séquence de leur parcours professionnel et, plus largement, de leur parcours de vie. Lointaines parentes des bourses du travail ayant émergé à la fin du XIXè siècle, elles s'en distinguent par leur indépendance vis-à-vis des syndicats et par la grande diversité des missions qu'elles se donnent. Dans cette partie, je m'appuierai sur mes propres recherches auprès de "Chœurs de fondeurs ", l'association des ex-salariés de Metaleurop-Nord.

Créée au lendemain de la signature du plan social, le 10 avril 2003, l'association poursuit, par d'autres 
moyens, la lutte engagée par l'intersyndicale 3 . Elle se dote, entre autres, d'une commission « accompagnement social» qui travaille en partenariat avec les intervenants institutionnels: Association nationale pour la formation professionnelle des adultes (AFPA), Agence nationale pour l'emploi (ANPE), antenne emploi (nom donné à la cellule de reclassement), Assedic, Conseil régional, Direction départementale du Travail, de l'Emploi et de la Formation professionnelle (DDTEFP)... Elle participe à la conférence des financeurs chargée des questions de la formation professionnelle ${ }^{4}$, et est présente dans les commissions de Validation des acquis de l'expérience, etc. Cette implication de l'association dans le suivi du plan social avait été négociée dès la signature du plan par l'intersyndicale, et d'autant mieux acceptée par les différents partenaires que l'employeur était défaillant et absent. Tant que l'antenne emploi reste active, soit jusqu'au 30 mars 2005, le travail d'accompagnement des ex-salariés est bénévole. En octobre de la même année, l'association obtient des subventions pour salarier deux personnes à mi-temps pendant un an sur des postes de «référents sociaux ». Elle embauche alors deux ex-Metaleurop, très actifs pendant la lutte, membres de l'intersyndicale et particulièrement engagés dans la commission « accompagnement social » depuis sa création. Un seul poste peut être financé d'octobre 2006 à avril 2007 et, depuis cette date, les référents travaillent à nouveau bénévolement.

Ni professionnels du reclassement, ni véritablement profanes, les référents sociaux sont des «professionnels de l'usine ». La première se décrit comme une «enfant de Penarroya» (entretien du 12 décembre 2006), du nom de l'ancien propriétaire de l'usine ${ }^{5}$. Ses collègues disent d'elle qu'elle est «née dans l'usine » (Corps de fondeurs). Ses deux

\footnotetext{
3 À propos de «Chœurs de fondeurs", voir le site de l'association: www.choeursdefondeurs.com; Corteel, Duhin, Hayem, 2007 et Corteel, 2009.

${ }^{4} \mathrm{La}$ conférence des financeurs réunissait l'Assedic, le Conseil régional, la Direction départementale du Travail, de l'Emploi et de la Formation professionnelle, l'Organisme paritaire collecteur agréé, l'Agence nationale pour l'emploi et l'Association de gestion du fonds pour l'insertion professionnelle des personnes handicapées. Les référents sociaux bénévoles de l'association des anciens salariés y siégeaient aussi.

5 Pour une rapide reprise de 1'histoire de 1'usine, voir Fajardie, 2003, pp. 20-27.
}

parents travaillaient à l'usine, elle a grandi dans une maison Penarroya, eu sa première expérience de travail à l'usine pendant les vacances scolaires. Elle y est entrée le 11 février 1971 et a travaillé toute sa carrière au service achat. Son collègue, moins bavard, est entré à l'usine le 2 novembre 1979. D'abord commercial, puis responsable de gestion dans un atelier de plomb, il a lui aussi travaillé pendant des années au service achat et milité au même syndicat qu'elle.

Tout en accompagnant chacun des anciens salariés, ils défendent une professionnalité collective. Ils s'appuient sur les valeurs de la profession et les réactualisent, permettant ainsi au collectif de perdurer et à la fierté de se maintenir, autant que faire se peut. "Le ciment qui a fait que l'association a pris, ce sont les conditions de travail» (entretien avec l'un des fondateurs de l'association, ancien contremaître, le 12 décembre 2006). Le travail des référents sociaux, encadré par l'association, les objectifs que celle-ci se donne et l'esprit dans lequel elle a été créée, s'inscrivent dans un esprit particulier : "Un accompagnement humain, social et la promotion des valeurs. [...] Les valeurs humaines, professionnelles : le travail en équipe autour d'un haut-fourneau, c'est quelque chose d'unique. La capacité des gens à affronter les difficultés, à faire tourner une usine dans un contexte un peu hostile de panne, coûte que coûte... c'est la solidarité, c'est une clairvoyance de la part des gens, fiers de faire cette production, de la faire ensemble. Une solidarité, un peu obligée parfois. » (entretien avec le vice-président de l'association, le 14 juin 2007). Cet ingénieur a lui aussi fait toute sa carrière à Metaleurop, où il s'est beaucoup impliqué dans les questions d'hygiène et de sécurité. L'accompagnement s'enracine donc explicitement dans l'expérience du travail à l'usine, et ce dans une double dimension.

D'une part, la dimension de la solidarité. Comme du temps des crises à l'usine - pannes, accidents -, face à la fermeture, on se serre les coudes; soutenir et accompagner les autres, c'est se soutenir soi-même. Issues du travail, des valeurs comme l'esprit d'équipe, la solidarité ou le courage guident le travail des référents sociaux et sont garantes d'un accompagnement équitable. L'équité exige que l'action des référents soit encadrée par l'association elle-même 
ou son bureau, selon les règles habituelles du fonctionnement associatif, faute de quoi on observe des dérives comme la réactivation d'amitiés ou d'inimitiés nourries du temps de l'usine.

D'autre part, les référents sociaux, ex-salariés euxmêmes, puisent dans leur expérience du travail à l'usine une connaissance presque intime du travail et des personnes que ne peuvent avoir les consultants des cabinets de reclassement ou les conseillers de l'ANPE. Pour l'avoir pratiqué et y avoir contribué eux-mêmes, ils connaissent le genre professionnel: «tout ce sur quoi les générations successives de professionnels ont buté, obligeant chacun, dans cette ouvre d'interprétation collective et singulière, à y mettre du sien. » (Clot, op. cit., p. 3) Ils appréhendent le travail de l'intérieur, d'un point de vue tout à la fois concret - la réalité de l'activité - et subjectifl'engagement de soi, les valeurs. Ce savoir fonde une expertise singulière qui leur permet d'intervenir en faveur d'ex-salariés dont l'expérience, les capacités ou les qualités seraient, sans eux, passées inaperçues.

Comme l'antenne emploi avec laquelle elle a travaillé tout le temps de son existence, l'association peut être envisagée comme un intermédiaire du marché du travail (Bessy et Eymard-Duvernay, 1997). Ancrée dans la profession, l'association propose une intermédiation originale qui se distingue de celle de l'antenne emploi et la complète.

\section{UN INTERMÉDIAIRE ORIGINAL}

L'antenne emploi fut mise en place rapidement. Elle rassemblait des consultants privés de deux cabinets différents, des personnels ANPE et AFPA réunis en plate-forme de services. L'association «chœurs de fondeurs »s'est impliquée dans le choix des cabinets privés et a même été à l'origine de ce montage original conduisant à la collaboration entre deux prestataires privés. Comme le montre Olivier Mazade, les consultants recrutés pour animer l'antenne emploi doivent faire face à une gestion du personnel «plus qu'optimisée » (2005, p. 205). En effet, le nombre de consultants présents et le temps dévolu à l'accompagnement sont calculés au plus juste pour atteindre les objectifs de rentabilité du prestataire privé, ce qui se répercute sur la nature et la qualité de l'accompagnement proposé. Mazade parle alors d'une dérive fonctionnelle qui délaisse l'aspect relationnel de l'accompagnement, pourtant nécessaire pour faire face au traumatisme que représente la fermeture. Le temps passé à identifier l'expérience singulière et les capacités des ex-salariés se trouve réduit à une peau de chagrin. De plus, les consultants qui ignorent tout du travail dans une fonderie ne peuvent s'appuyer sur leurs propres connaissances pour aider les personnes à identifier et à traduire les compétences et qualifications construites au sein du collectif de travail antérieur. Dans ces conditions, le travail d'exploration de l'expérience, de traduction dans des termes utiles pour l'avenir et de transcription écrite se trouve à la charge des ex-salariés isolés dans le face-à-face avec leur consultant. C'est un travail difficile et douloureux, en raison du retour nécessaire sur un passé qui passe mal, mais aussi à cause de la réflexivité et de la maîtrise de l'écrit qu'il requiert; compétences que n'ont pas nécessairement cultivées les fondeurs dans leur vie passée. Bruggeman et al. ont montré l'importance et le sens du collectif dans la construction des compétences (2005, p. 74). L'intervention des référents sociaux et de l'association des anciens salariés, par leur connaissance fine du genre professionnel, peut apporter une expertise singulière pour aider à l'exploration de l'expérience. Cette intervention permet de redonner à l'ancien collectif de travail un sens et une valeur pour envisager l'avenir. D'autres acteurs peuvent également appuyer le processus. L'Agence régionale d'amélioration des conditions de travail a, quant à elle, permis de traduire l'expérience et de prendre un peu de hauteur en identifiant, par exemple, des compétences en matière de « gestion du risque ».

Par ailleurs, les consultants, pressés par une gestion du personnel optimisée ainsi que par les objectifs auxquels ils sont soumis en termes de retour à l'emploi ou de solution identifiée (invalidité, reconnaissance du statut de travailleur handicapé...), ont tout intérêt à recourir à des canaux de recrutement et des techniques de recherche d'emploi standardisés : rédaction d'un curriculum vitae, réponse aux petites annonces publiées sur support papier ou sur internet, etc. Bessy et Marchal (2009) ont montré que ces canaux de recrutement et ce mode de prospection 
pénalisent plus particulièrement les ouvriers non qualifiés ayant une (assez) longue expérience professionnelle. Celle-ci est en effet difficile à mettre en valeur sur un $\mathrm{CV}$ dans la mesure où ce dernier met en avant des ressources qui relèvent de critères très généraux comme les diplômes, les titres ou les différentes fonctions occupées. Ressources dont les fondeurs de Metaleurop-Nord ne disposent pas ou peu. De plus, ces canaux de recrutement multiplient les épreuves: tests, entretiens répétés... qui ne permettent pas davantage au candidat de faire valoir son expérience singulière et qui constituent autant d'obstacles difficiles à surmonter pour des personnes déjà fragilisées par la fermeture. Ainsi, la cellule de reclassement constitue un support intéressant pour les ex-salariés disposant déjà des ressources mobilisables sur le marché, dans la mesure où elle les aide à mettre ces ressources en valeur, mais l'intervention d'autres acteurs peut s'avérer nécessaire pour accompagner d'autres salariés moins bien dotés.

Les référents sociaux, on l'a vu, réalisent un accompagnement social et professionnel gouverné par les valeurs professionnelles héritées de l'expérience de travail à l'usine. Ces valeurs sont partagées et mises en œuvre par nombre d'ex-salariés qui ne manquent pas de faire circuler les informations : "tous les exsalariés en CDI nous font connaître les postes disponibles dans leur entreprise. » (entretien avec les référents sociaux, 18 octobre 2006), "quelqu'un voit un profil de travail qui peut intéresser un ancien de Metaleurop, il va nous appeler. » (entretien avec les référents sociaux, 12 décembre 2006) Lors de séances d'observation, nous avons pu assister à ce type de scènes, soit au quotidien dans le bureau des référents, soit dans des circonstances plus exceptionnelles comme lors des Assemblées générales de l'association. Ainsi, les référents sociaux transforment l'ancien collectif de travail en un réseau alimenté par le réseau de chacun et mobilisable par tous les membres de l'association. Granovetter (2000) a montré l'importance des réseaux, et en particulier celle des liens faibles - les relations professionnelles - pour trouver un emploi. Il indique également que les personnes les moins dotées en qualification et occupant ou ayant occupé un emploi subalterne sont aussi souvent celles qui disposent d'un réseau peu étendu. Par voie de conséquence, elles se trouvent doublement pénalisées quand il leur faut chercher un nouvel emploi. Les travaux récents de Bessy et Marchal (op. cit.) s'inscrivent dans cette approche et insistent sur le fait que le recrutement via le réseau, en procédant par le bouche à oreille, permet de mettre en avant une expérience riche et singulière et évite aux candidats des épreuves nombreuses et répétées. De ce fait, contrairement au marché, le réseau donne plus de chances de retrouver un emploi aux ouvriers non qualifiés et âgés qui « restent sur le carreau » des fermetures de sites industriels comme celui de Metaleurop. Par cette activité de constitution et d'animation d'un réseau qui permet à chacun de profiter des liens faibles et des contacts des autres, l'association et ses référents sociaux viennent ainsi corriger, au moins en partie, certaines inégalités sur le marché du travail auxquelles sont confrontés les ex-salariés.

L'antenne emploi peut représenter un support intéressant pour les ex-salariés qui s'engagent dans des parcours de formation afin d'obtenir des ressources mobilisables sur le marché. Certains ex-salariés ont en effet saisi l'occasion de la fermeture pour entamer une formation, soit dans le prolongement de leur ancien travail, soit en totale rupture avec leur ancienne profession, comme pour ceux qui sont devenus aidessoignants. Ces formations sont d'autant plus nécessaires dans le contexte de la disparition de l'usine et de la profession. Elles leur ont permis d'obtenir un titre, une certification, parfois même un diplôme. Les exsalariés de Metaleurop ont souvent été aiguillés dans cette voie par l'antenne emploi. Mais l'association a également joué un rôle essentiel grâce à sa connaissance fine de l'usine, du travail et des personnes.

En effet, contrairement aux idées reçues, les salariés de Metaleurop ont bénéficié de nombreuses formations tout au long de leur parcours professionnel à l'usine, comme le souligne l'ancien responsable de la formation de Metaleurop ${ }^{6}$ : «J'ai eu de la chance, je suis tombé sur un groupe [Metaleurop] qui a été parmi les dix premiers en France pour son plan de formation. Il y avait une idée de la formation.

\footnotetext{
${ }^{6}$ Après un parcours au sein du groupe Nickel-Penarroya-Mokta, cet ancien chimiste s'est découvert une passion pour la formation qui l'a conduit un peu partout dans le monde, en particulier en Afrique. Il s'est ensuite fixé dans le Nord-Pas-de-Calais à son arrivée à Metaleurop Nord en 1986. Au moment de la fermeture, il était à un an de la retraite.
} 
Comme il y avait pas mal de démarrages d'unités, on a su vendre l'idée qu'on n'a pas le droit de démarrer une unité sans former le personnel.» (entretien du 14 juin 2007) On peut évoquer, à titre d'exemple, le parcours de Jean-Pierre: "Quand je suis entré à Metaleurop, c'était l'époque où ils mettaient en place les programmes de formation. C'était une chance. Comme j'aime bien apprendre, étudier, j'ai fait la formation tout au long de ma carrière, dans tous les domaines. J'ai démarré comme balayeur, puis j'ai fait un peu de tout.» (entretien du 12 décembre 2006) Au moment de la fermeture, vingt huit ans après son entrée dans l'usine à l'âge de 21 ans, sans aucun diplôme, Jean-Pierre était contremaître. Rarement diplômantes, ces formations dispensées du temps de l'usine ne laissent pas de trace visible sur un curriculum vitae. Dans le cadre de la conférence des financeurs, qui réunissait l'ensemble des acteurs ayant en charge la formation ainsi que les référents sociaux représentant l'association, ces derniers ont été particulièrement actifs. Ils ont soutenu des dossiers qui, pour un observateur extérieur, ne présentaient pas forcément toutes les chances de réussite. Leur connaissance du travail et du parcours des personnes à l'usine leur a conféré une légitimité particulière pour juger des capacités des anciens salariés, à la fois vis-à-vis des financeurs et de leurs collègues : "Notre rôle c'est de dire de quoi les gens sont capables. Quand on savait qu'une personne pouvait réussir [une formation] on l'a dit, et ça a été pris en compte, et ils ont réussi.» (entretien avec les référents sociaux, 18 octobre 2006) Cette légitimité leur permet de rendre lisibles les capacités et compétences des personnes et de les traduire afin de rendre possible une nouvelle séquence professionnelle, grâce à l'acquisition de nouvelles ressources, qui s'appuie sur la prise en compte de l'expérience. Ils ouvrent ainsi la voie à des procédures d'évaluation connectées au contexte de travail antérieur et apportent des informations et des critères de jugement moins standardisés et plus contextualisés, susceptibles de donner leur chance à des personnes qui auraient été pénalisées en leur absence.

De même, s'agissant des procédures de Validation des acquis de l'expérience (VAE), les référents sociaux de l'association ont soutenu leurs anciens collègues contre l'avis d'experts présents à la commission des financeurs, des universitaires notamment : «La VAE, la première réunion, ça s'est très mal passé. Des salariés qui avaient au départ un $C A P^{7}$ voulaient valider un $B T S^{8}$; au départ, les universitaires de la commission ont bloqué, ils ont dit non, on a dit "si, ils sont capables" et ils l'ont tous eu haut la main.» (entretien avec les référents sociaux, 18 octobre 2006) Avec l'aide d'autres exsalariés, ils ont accompagné les candidats à la VAE dans la constitution de leur dossier, en particulier en ce qui concerne la description détaillée des contenus des postes occupés. Fins connaisseurs des produits, des techniques et des procédés de fabrication ainsi que des postes, les référents sociaux et le réseau des anciens salariés apportent une expertise utile à leurs collègues pour franchir ce passage obligé de tout dossier de VAE. Dans cette procédure, l'association représente un support collectif supplémentaire à la disposition de tout ex-salarié souhaitant s'engager dans cette démarche.

Enfin, les référents sociaux poursuivent leur activité au-delà du temps dévolu à l'antenne emploi et continuent un travail d'accompagnement fort nécessaire, comme le soulignent Aubert et Beaujolin-Bellet (2004) $)^{9}$. En effet, l'étude des dispositifs d'accompagnement des salariés licenciés montre qu'une personne sur deux seulement retrouve un emploi à l'issue de la période de reclassement, quel que soit le statut d'emploi (DARES, 2006). Malgré l'implication des référents sociaux, les résultats en termes de retour à l'emploi des anciens salariés de Metaleurop, à l'issue de la période où l'antenne emploi a officié, ne sont pas spectaculaires. Outre la situation économique du territoire, qui ne doit pas être oubliée quand on regarde ces chiffres, il faut signaler qu'au moment de la fermeture, la moyenne d'âge du personnel de l'usine est relativement élevée, puisqu'elle est de 48 ans. L'âge, l'ancienneté dans l'entreprise ( 20 ans en moyenne) ainsi que la lutte longue et dure menée par les anciens salariés constituent des facteurs d'«inemployabilité », comme le soulignent Dietrich, Jouvenot et Lenain (2007). À la

\footnotetext{
${ }^{7}$ Certificat d'aptitude professionnelle, diplôme de niveau V.

${ }^{8}$ Brevet de technicien supérieur, diplôme de niveau III.

${ }^{9}$ Suivi à long terme absent des dispositifs actuels, comme le déplorent Aubert et Beaujolin-Bellet (2004).
} 
fin de l'antenne emploi, au 30 mars 2005, soit deux ans après la fermeture, sur les 679 personnes à reclasser, 344 sont en emploi au sens utilisé par la DARES $(2006)^{10}$ soit $51,2 \%$ des personnes inscrites à l'antenne emploi, ce qui est inférieur à la moyenne nationale de 2004 (56,0\% : soit les personnes ayant adhéré à une cellule de reclassement en 2004 à la sortie du dispositif). Cependant, le taux de CDI est, quant à lui, supérieur aux chiffres nationaux de 2004 : $36 \%$ pour Metaleurop contre 28,7\%. En juin 2007, soit quatre ans après la fermeture, ces chiffres n'ont pas cessé d'augmenter puisque le nombre de personnes en emploi atteint 449 personnes, soit $66,9 \%$, dont 323 en CDI, soit $48,1 \%$ des personnes initialement inscrites à l'antenne emploi. Par ailleurs, l'installation de nouvelles entreprises sur le site, à

${ }^{10} \mathrm{CDI}, \mathrm{CDD}$, intérim et création d'entreprise. l'automne 2007 et courant 2008, permet d'envisager la poursuite de l'augmentation de ces chiffres. En juillet 2009, d'après l'AFPA : « sur les 60 personnes non reclassées, 37 ont trouvé une solution stabilisée.» (dont treize en CDI, une création d'entreprise, et quatre invalidités reconnues) ${ }^{11}$.

«L'accompagnement devient de plus en plus important parce que les gens ont des situations financières et psychologiques pas bonnes du tout. Il faut essayer de trouver toute solution pour qu'ils soient bien dans leur tête pour reprendre le travail » (entretien avec les référents sociaux, 13 juin 2007). Outre l'animation et l'exploitation du réseau, ils continuent d'inventer des médiations en participant, avec l'ANPE et

${ }^{11}$ Communiqué de l'AFPA du 20 juillet 2009.

Tableau 1

Situation professionnelle des ex-salariés de Metaleurop

\begin{tabular}{|c|c|c|}
\hline Situation au : & $30 / 03 / 2005$ & $13 / 06 / 2007$ \\
\hline Création d'entreprise & 18 & 23 \\
\hline CDI & 243 & 323 \\
\hline CDD & 48 & 58 \\
\hline Intérim & 35 & 45 \\
\hline Sous-total en emploi & 344 & 449 \\
\hline Demandeurs d'emploi & 216 & 77 \\
\hline Formation + de 6 mois & 29 & 7 \\
\hline Dispensé de recherche d'emploi (*) & 28 & 82 \\
\hline Cessation d'activité ; amiante & - & 8 \\
\hline Retraités & - & 15 \\
\hline Problème de santé $(* *)$ & 30 & - \\
\hline Invalidité et longue maladie & - & 19 \\
\hline Aucune information & 17 & 4 \\
\hline Non inscrit à l'antenne emploi & 8 & - \\
\hline Décès & 7 & 18 \\
\hline TOTAL & 679 & 679 \\
\hline
\end{tabular}

Source : Association « Chœurs de fondeurs », tableau de situation des ex-salariés. Le tableau ne correspond pas exactement à celui présenté par « Chœurs de fondeurs ». L'ordre d'apparition des critères et leur regroupement ont été révisés pour permettre une comparaison avec les chiffres de la DARES, les personnes en emploi au sens de la DARES comprenant : les CDI, les CDD (respectivement contrat à durée indéterminée et contrat à durée déterminée), l'intérim et la création d'entreprise.

(*) : Certaines personnes sont dispensées de recherche d'emploi par l'ANPE, en particulier lorsqu'elles approchent de l'âge de la retraite. (**) : Les personnes regroupées dans cette catégorie dans la première colonne ont ensuite bénéficié d'une cessation anticipée d'activité pour exposition à l'amiante, d'une reconnaissance de travailleur handicapé ou encore du statut d'invalide. Certaines sont décédées. 
la Mission Locale, à des processus de présélection à l'embauche et en organisant des séances de simulation d'entretien dans le cadre de la réindustrialisation du site de l'usine. "Le recrutement est étalé jusque fin 2008, voire début 2009, suivant les nouvelles installations. On a été amenés à jouer un rôle à chaque étape. Nous on a convoqué toutes les personnes en situation précaire (c'est-à-dire les personnes en $C D D$, en intérim, en formation ou demandeurs d'emploi). Ca fait quand même presque 200 personnes. On a des réunions prévues avec les DRH pour faire le point 》 (entretien avec les référents sociaux, 13 juin 2007). La participation de l'association au recrutement organisé par les entreprises qui s'installent sur le site de Metaleurop, après destruction de l'usine et dépollution, résulte de l'implication de l'association dans le choix du repreneur du site. Son action s'inscrit dans une convention de type réseau (Eymard-Duvernay, Marchal, 1996) où l'évaluateur est un médiateur qui met en relation des candidats et des entreprises sur un territoire et se porte garant des candidats. Là encore, l'expérience du genre professionnel se rapportant à la fonderie et au métier de fondeur, la connaissance des personnes au travail et de leur situation personnelle actuelle fondent le jugement des référents sociaux sur la qualité des candidats.

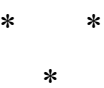

L'activité des référents sociaux, sous l'égide de l'association des anciens salariés, s'inscrit dans un temps ouvert. Si elle a vocation à s'arrêter un jour, le terme n'est pas fixé. Moitié salariée, moitié bénévole, leur action n'est soumise ni à une gestion du personnel optimisée, ni à des objectifs chiffrés. Alors que les cellules de reclassement, intermédiaires privés, agissent selon un modèle de marché et que Bureau et Marchal mentionnent, à propos des intermédiaires privés, "le marché n'a pas de cour» (2005, p. 21), les référents sociaux déclarent : «nous travaillons avec notre cour et avec notre âme.» (entretien du 18 octobre 2006) Ils s'inscrivent dans une autre logique, une autre rationalité que celle des intermédiaires privés, une logique qui prend ses racines dans la profession disparue et construit un rapport au passé dans le processus même de réélaboration de l'expérience. «Professionnels de l'usine », ils réactualisent les valeurs professionnelles de solidarité et de courage et mettent au service de leurs collègues leur expertise singulière issue de la connaissance presque intime du genre professionnel passé. Ils ne s'opposent pas aux autres intermédiaires et ne s'y substituent pas non plus mais travaillent avec eux, en tentant d'apporter une contribution originale. Ce faisant, l'association et ses référents se présentent comme un "espace de médiation» (Guérin, 2002).

Un espace de médiation entre les anciens salariés, les professionnels du reclassement et les autres intermédiaires du marché du travail d'une part. En effet, intermédiaire reconnu par les autres partenaires institutionnels impliqués dans le suivi du plan de sauvegarde de l'emploi, ils portent la voix de leurs collègues dans les instances comme la conférence des financeurs et font valoir des qualités qui ne sont pas toujours lisibles dans les termes des conventions à l'œuvre. Ils ouvrent la voie à des procédures d'évaluation connectées au contexte de travail antérieur. En retour, ils aident leurs collègues à transcrire leur expérience dans les termes attendus par les intermédiaires traditionnels du marché du travail.

Un espace de médiation entre les anciens salariés d'autre part. L'association offre aux anciens salariés un espace de rencontre et de dialogue. Elle permet que se côtoient des personnes qui ne s'étaient jamais parlées du temps où l'usine fonctionnait et où les clivages de classe, notamment, instauraient des compartiments relativement étanches. Face à la fermeture, comme lors des accidents mortels des années 90, les anciennes lignes de fracture disparaissent et la communauté se (re)soude pour affronter cet événement violent et traumatique. Ce collectif réinventé réaffirme la force des liens faibles et initie la formation d'un réseau au service de la poursuite des parcours (professionnels) des uns et des autres. L'association s'appuie sur les liens sociaux hérités du travail et de la lutte contre la fermeture et, modestement, tisse et entretient des liens entre les personnes. Les référents sociaux constituent un nœud essentiel de ce réseau en collectant offres et demandes et orientant ensuite vers tel ou tel en fonction des besoins. 
Un espace de médiation entre le passé et l'avenir. Là où les cellules de reclassement invitent souvent à tourner la page et considèrent l'ancien collectif de travail comme un obstacle, venant réaffirmer l'inutilité de l'expérience brutalement assénée par la fermeture, l'association permet au contraire de donner un sens et une utilité nouvelle à l'expérience collective et à la profession disparue. La fermeture, événement biographique partagé, invite à réexaminer le passé et l'avenir ; grâce à l'association, ce processus s'engage collectivement, évitant la solitude et l'isolement: «les gens se sont parlés d'homme à homme, c'est-àdire pas comme premier fondeur mais quelque chose de très fort dans la communication, une auto-analyse psychanalytique. Chacun s'est mis à parler et en parlant on a un peu abandonné cette culpabilité, on était plus forts, on n'était plus seuls. » (entretien avec un ancien ingénieur, co-fondateur et membre actif de l'association, le 11 décembre 2006) Les membres de l'association et les référents y puisent des ressources pour l'avenir de chaque ancien salarié qui les sollicite. Ils permettent ainsi à ces derniers de ne pas abandonner la part de soi engagée dans le collectif de travail et dans l'usine aujourd'hui fermée et de défendre la dignité et l'honneur en jeu dans le rapport au travail, qu'il soit envisagé sous l'angle de la profession (Hughes, op. cit.) ou du don (Mauss, [1923-24] 2007 ; Godbout, 2007 ; Malsan, 2007).

À l'exemple de «Chœurs de fondeurs », les associations d'anciens salariés constituent un objet d'étude qui ouvre des pistes prometteuses et qu'il est urgent de creuser au vu de la conjoncture financière mondiale qui entraîne la chute et la fermeture de nombreuses entreprises. Par leur action, des associations comme "Chœurs de fondeurs » désignent un possible, une autre manière d'envisager les situations de fermeture et l'accompagnement proposé aux personnes licenciées, qui complète utilement l'intervention des intermédiaires traditionnels. Pourtant, dans des cas comme Job ou Moulinex, où les associations existent et où, visiblement, leur rôle dépasse celui d'une amicale des anciens, les sociologues omettent de leur accorder le statut d'objet de recherche. Y aurait-il, dans cette absence, quelque chose à comprendre de l'espace professionnel des sociologues eux-mêmes ?

\section{Bibliographie}

Aubert J.-P., Beaujolin-Bellet R. (2004), « Les acteurs de l'entreprise face aux restructurations: une délicate mutation », Travail et Emploi, $\mathrm{n}^{\circ} 100$, pp. 99-112.

Bessy Ch., Eymard-Duvernay F. (dir.) (1997), Les intermédiaires $d u$ marché du travail, Cahiers du Centre d'Études de l'Emploi, Paris, PUF.

Bessy Ch., Marchal E. (2009), «Le rôle des réseaux et du marché dans les recrutements. Enquête auprès des entreprises », Revue Française de SocioEconomie, 1 ${ }^{\text {er }}$ semestre, pp. 121-146.

Bruggeman F., Paucard D., Tuchszirer C. (2005), Privé-Public: une analyse des cellules de reclasse- ment par l'examen des jeux d'acteurs, Étude réalisée pour la DGEFP, Document de travail de l'IRES, $\mathrm{n}^{\circ} 05-03,138 \mathrm{p}$.

Bureau M.-C., Marchal E. (Éds.) (2005), Au risque de l'évaluation: salariés et candidats soumis aux aléas du jugement, Villeneuve d'Ascq, Presses Universitaires du Septentrion.

Clot Y. (2003), « Le développement du collectif : entre l'individu et l'organisation du travail », communication au Colloque de Cerisy, septembre, $14 \mathrm{p}$.

Corteel D. (2009), « Lutter pour la reconversion d'un site industriel et la dignité des hommes qui l'ont fait vivre. Analyse d'expériences menées par les ex- 
salariés de Metaleurop-Nord. », in J.-Y. Causer, J.-P. Durand et W. Gasparini (dir.), Les identités au travail. Analyses et controverses, Toulouse, Octarès, pp. 113-122.

Corteel D., Duhin A., Hayem J. (2009) « Metaleurop: dal difensivo al creativo. Cronaca e analisi di una lotta singolare », in Valerio Romitelli (dir.), Fuori della società della conoscenza: ricerche di etnografia del pensiero, Infinito, Roma, pp. 265-280.

Corteel D., Zimmermann B. (2007), «Capacités et développement professionnel », Formation Emploi, $\mathrm{n}^{\circ} 98$, avril-juin.

DARES (2006), « Les dispositifs publics d'accompagnement des restructurations en $2005 »$, Premières synthèses, $\mathrm{n}^{\circ} 37.2$.

Dietrich A., Jouvenot C., Lenain M.-C. (2007), «L'employabilité sous conditions. Le cas Metaleurop », Éducation permanente, $\mathrm{n}^{\circ} 170$, pp. 131-141.

Eymard-Duverney F., Marchal E. (1997), Façons de recruter : le jugement des compétences sur le marché du travail, Paris, Métailié.

Fajardie F. (2003), Metaleurop, Paroles ouvrières, Paris, Mille et une nuits.

Gaulin M.-J. (2005), Metaleurop : une crise locale aux causes internationales et aux conséquences nationales, mémoire de maîtrise, université Paris I.

Godbout J.-T. (2007), Ce qui circule entre nous. Donner, recevoir, rendre, Paris, Seuil.

Granovetter M. (2000), Le marché autrement. Les réseaux dans l'économie, Paris, Desclée de Brouwer.

Guérin I. (2000), «Usage des minima sociaux et conversion des droits formels en droits réels : le rôle des espaces de médiation », in A. Alcouffe, B. Fourcade, J.-M. Plassard, G. Tahar (coord.), Efficacité versus équité en économie sociale, $\mathrm{XX}^{\mathrm{e}}$ journées de l'AES, Tome 2, Paris, L'Harmattan, pp. 350-360.
Hughes Everett C. (1996), Le regard sociologique, Paris, Éditions de l'École des Hautes Études en Sciences Sociales.

Leclerc-Olive M. (1998), «Les figures du temps biographique », Cahiers Internationaux de Sociologie, $\mathrm{n}^{\circ} 104$, pp. 97-120.

Le Quentrec Y., Benson S. (2005), Un Job pour la vie, les salariés de Job en lutte (1995-2001), Paris, Syllepse.

Linhart D., Rist B., Durand E. (2002), Perte d'emploi, perte de soi, Ramonville Saint Agne, Erès.

Maison des Hommes et des Techniques (2007), Fermetures de boîtes... et après?, Nantes, Éditions du Centre d'histoire du travail.

Malsan S. (2001), Les filles d'Alcatel. Histoire d'une reconversion industrielle, Toulouse, Octarès.

Malsan S. (2007), «Licenciements collectifs : le prix d'une dette symbolique », Revue du Mauss, $\mathrm{n}^{\circ} 29$, pp. 150-176.

Mauss M. (2007) [1923-1924], Essai sur le don, formes et raisons de l'échange dans les sociétés archaïques, Paris, PUF.

Mazade O. (2005), "Cellules de reclassement et individualisation du traitement du chômage. Le cas de Metaleurop Nord et des Houillères du Nord», Revue de l'IRES, n 47 , pp. 195-214.

Rossignol S. (2008), Notre usine est un roman, Paris, La Découverte.

Roupnel-Fuentes M. (2007), Une rupture totale, le licenciement massif des salariés de Moulinex, thèse de doctorat en sociologie, École des Hautes Études en Sciences Sociales.

Salais R., Storper M. (1993), Les mondes de production : enquête sur l'identité économique de la France, Paris, Éditions de l'EHESS.

Tuchszirer C. (2005), « Le reclassement des salariés licenciés pour motif économique: responsabilité sociale de l'entreprise ou de la collectivité publique ? », Revue de l'IRES, n 47, pp. 157-173. 


\title{
Résumé
}

\section{Fermetures d'usines : les associations d'anciens salariés comme espaces de médiation originaux}

\author{
Delphine Corteel
}

Comment des salariés confrontés à la fermeture de leur usine peuventils être accompagnés en vue de la poursuite de leur parcours professionnel ? En particulier, quel est le rôle possible du collectif professionnel antérieur - obstacle ou ressource? Cet article explore la piste ouverte par l'expérience originale du travail d'accompagnement réalisé par l'association des anciens salariés de Métaleurop-Nord. Les membres de l'association, "professionnels de l'usine », ne se substituent pas aux professionnels du reclassement, mais travaillent avec eux et apportent une expertise singulière issue de leur expérience du travail et de l'usine. Ils réinventent le collectif et le mobilisent pour soutenir les projets professionnels des anciens salariés. Ce faisant, ils permettent de redonner un sens et une utilité à la profession aujourd'hui disparue, de réactiver les réseaux et les liens constitués au moment où l'usine fonctionnait, au service des avenirs professionnels de ceux qui les sollicitent.

\section{Mots clés}

Reclassement professionnel, accompagnement professionnel, projet professionnel, association Journal of Economic Literature: J 44, M 51 